\title{
Diagnostic accuracy of the T-MACS decision aid with a contemporary point of care troponin assay
}

SHORT TITLE:

T-MACS with point of care troponin

AUTHORS:

Richard Body; ${ }^{\text {a, b, c }}$ Malak Almashali; ${ }^{c}$ Niall Morris;; $;$ b Phil Moss; ${ }^{d}$ Heather Jarman; ${ }^{d}$ Andrew Appelboam ; Richard Parris; ${ }^{\mathrm{f}}$ Louisa Chan; ${ }^{\mathrm{g}}$ Alison Walker; ${ }^{\mathrm{h}}$ Mark Harrison; Andrea Wootten;j Garry McDowell

\section{Affiliations:}

a: Emergency Department, Central Manchester University Hospitals NHS Foundation Trust, Oxford Road, Manchester, M13 9WL, United Kingdom

b: Cardiovascular Sciences Research Group, The University of Manchester, Manchester Academic Health Science Centre, Oxford Road, Manchester, M13 9PL, United Kingdom c: School of Healthcare Science, Manchester Metropolitan University, Oxford Road, Manchester, United Kingdom

d: Emergency Department, St. George's NHS Foundation Trust,

e: Emergency Department, Royal Devon \& Exeter Hospital NHS Foundation Trust

f: Emergency Department, Bolton NHS Foundation Trust

g: Emergency Department, Hampshire Hospitals NHS Foundation Trust

h: Emergency Department, Harrogate and District NHS Foundation Trust

i: Emergency Department, Northumbria Healthcare NHS Foundation Trust

j: Emergency Department, Arrowe Park Hospital, Wirral University Teaching Hospital NHS Foundation Trust

\section{Corresponding author and address:}

Prof Richard Body

Professor and Honorary Consultant in Emergency Medicine

Emergency Department, Manchester Royal Infirmary, Oxford Road, Manchester, M13 9WL, United Kingdom

Email: richard.body@manchester.ac.uk

Telephone: 00447880712929 
Key words: Acute Coronary Syndromes; Clinical Decision Rules; Cardiac Troponin; Sensitivity and Specificity

Word count: 3,580 words; Abstract word count: 250 words

\section{Declarations}

Richard Body: Speaker fees from Singulex; Roche (consultancy, research grant); Abbott Point of Care (speaker fee, research grant); FABPulous BV (consultancy); Alere (donation of reagents for research)

\section{Funding sources and sponsorship}

This study was funded by a research grant from Abbott Point of Care. The study was sponsored by Manchester University NHS Foundation Trust.

The Corresponding Author has the right to grant on behalf of all authors and does grant on behalf of all authors, an exclusive licence (or non exclusive for government employees) on a worldwide basis to the BMJ Publishing Group Ltd and its Licensees to permit this article (if accepted) to be published in HEART editions and any other BMJPGL products to exploit all subsidiary rights 


\section{Abstract}

\section{Objectives}

The rapid turnaround time of point of care (POC) cardiac troponin (cTn) assays is highly attractive for crowded Emergency Departments (EDs). We evaluated the diagnostic accuracy of the Troponin-only Manchester Acute Coronary Syndromes (T-MACS) decision aid with a POC cTn assay.

\section{Methods}

In a prospective diagnostic accuracy study at 8 EDs, we included patients with suspected acute coronary syndromes (ACS). Blood drawn on arrival and 3 hours later was analysed for POC cTnl (iStat, Abbott Point of Care). The primary outcome was a diagnosis of ACS, which included both an adjudicated diagnosis of acute myocardial infarction (AMI) based on serial laboratory cTn testing and major adverse cardiac events (death, AMI or coronary revascularisation) within 30 days.

\section{Results}

Of 716 patients included, 105 (14.7\%) had ACS. Using serial POC cTnl concentrations over 3 hours could have 'ruled out' ACS in 198 (31.2\%) patients with a sensitivity of 99.0\% (95\% Cl 94.4-100.0\%) and negative predictive value 99.5\% (95\% Cl 96.5 - 99.9\%). No AMIs were missed. T-MACS 'ruled in' ACS for 65 (10.4\%) patients with a positive predictive value of $91.2 \%(95 \% \mathrm{Cl} 82.1-95.9 \%)$ and specificity $98.9 \%(97.6-99.6 \%)$.

\section{Conclusion}

With a POC cTnl assay, T-MACS could 'rule out' ACS for approximately one third of patients within 3 hours while 'ruling in' ACS for another $10 \%$. The rapid turnaround time and portability of the POC assay make this an attractive pathway for use in crowded EDs or urgent care centres. Future work should also evaluate use in the pre-hospital environment. 


\section{Background}

Chest pain is the second most common reason for emergency hospital admission [1]. However, as the prevalence of acute coronary syndromes (ACS) in those who are admitted on suspicion of that diagnosis is less than $20 \%[2,3]$, many hospital admissions could be avoided with improved diagnostic technology. It may now be possible to 'rule out' ACS following a single blood test in the Emergency Department (ED) for some patients. This can be achieved, for example, by using the limit of detection (LoD) of a high sensitivity cardiac troponin (hs-cTn) assay as a 'rule out' threshold [4,5], the History, electrocardiogram (ECG), Age, Risk factors and Troponin (HEART) score [6] or the Troponin-only Manchester Acute Coronary Syndromes (T-MACS) decision aid [2,7].

These algorithms, however, currently rely on the use of central laboratory troponin assays, which have a relatively long turnaround time (TAT). The target TAT is 60 minutes from receipt of the sample in the laboratory [8] but this does not account for pre-analytical (including time to collect and transport samples) and post-analytical factors. The use of near-patient cardiac troponin testing could help to reduce overall TAT. Because contemporary point of care (POC) cardiac troponin assays do not have the same sensitivity and precision as laboratory assays, diagnostic algorithms must be specifically validated with these assays before clinical use.

The T-MACS decision aid could be used to both 'rule in' and 'rule out' ACS by using an algorithm (derived by logistic regression) to calculate the probability of ACS using basic data about a patient's symptoms, signs, electrocardiogram and cardiac troponin (cTn) concentrations. To date, T-MACS has only been validated using high-sensitivity [7] and contemporary [9] central laboratory-based cTn assays. However, successful validation with a point of care (POC) cTn assay would reduce turnaround time, helping to unburden crowded EDs. Because contemporary POC cTn assays generally have 
inferior sensitivity and precision to central laboratory assays, we recognised that serial sampling may be required in order to achieve adequate diagnostic accuracy. However, given that the TAT of POC cTn assays is as little as 10-15 minutes, serial sampling over 3 hours could still facilitate rapid decision making. Importantly, this would enable rapid diagnosis even in situations where central laboratory cTn assays are not immediately available. We therefore aimed to prospectively validate T-MACS generated with a contemporary POC cTn assay, using (a) a single admission blood sample; and (b) two samples drawn 3 hours apart.

\section{Methods}

\section{Design and setting}

We undertook a multi-centre, prospective diagnostic test accuracy study at eight EDs in England (see Supplementary Appendix for details of each site). The study was approved by the National Research Ethics Service (reference 14/NW/1344), was sponsored by Manchester University NHS Foundation Trust and was undertaken in full compliance with the Declaration of Helsinki and according to the principles of Good Clinical Practice. The study was prospectively registered on the National Institute for Health Research (NIHR) portfolio (reference UK CRN 18000). All participants provided written informed consent.

\section{Study participants}

We included adults (aged $>18$ years) who presented to the ED with pain, discomfort or pressure in the chest, epigastrium, neck, jaw or upper limb without an apparent non-cardiac source [10], which the treating physician believed warranted investigation for possible ACS. We excluded patients 
whose peak symptoms had occurred $>12$ hours prior to presentation at the ED, patients with unequivocal evidence of ST elevation myocardial infarction requiring referral for immediate revascularisation, patients with another medical condition requiring hospital admission and patients who lacked the mental capacity to provide written informed consent. To expedite recruitment and avoid delays to blood sampling and clinical care, the initial blood samples for this study could be drawn at the time of arrival in the ED and at the same time as routine clinical samples without delay, with written consent obtained thereafter. In the event that written consent could not be obtained, samples were discarded and patients were not included in the study. Because of logistical, training and governance requirements, we included a convenience sample dictated by the availability of research nurses or study investigators. Sites were opened in phases with the first site commencing recruitment on $9^{\text {th }}$ February 2015 and the final site completing recruitment on $25^{\text {th }}$ October 2016.

\section{Data collection}

The treating clinician and study nurse recorded comprehensive clinical data at the time of inclusion using a bespoke case report form, in accordance with contemporary international standards. These data included details of: the presenting complaint; previous medical history; medication history; social history (including alcohol intake and tobacco use); family history of ischaemic heart disease; findings on physical examination; 12-lead ECG findings (including the presence or absence of dynamic ECG changes such as T wave inversion or ST segment depression); medications received during the active study phase; disposition; findings of relevant laboratory tests and medical imaging. The variables required for calculation of T-MACS were recorded by the treating clinician. 'Worsening' angina' was determined to be present or absent at the discretion of the clinician, but included patients with known angina or those with symptoms suspicious for new angina who had symptoms with increasing frequency, intensity or duration, or with less provocation (e.g. exertion) than usual. 
The interobserver reliability of all constituent variables in T-MACS has previously been established, and all variables had a kappa score $>0.6$ [2]. Interobserver reliability was not re-evaluated in this study.

In this observational study, patients were treated according to local guidelines, but in order to be selected for the study all sites were required to confirm that local practices were consistent with the guidance issued by the National Institute of Health and Care Excellence [11] and the European Society of Cardiology [12].

\section{Laboratory analyses}

Patients underwent venepuncture at the time of arrival in the ED and 3 hours (+/- 30 minutes) later. Whole blood (collected in lithium heparin vials) was analysed for cardiac troponin using the i-Stat assay (Abbott Point of Care, New Jersey, 99th percentile 80ng/L, LoD 20ng/L, co-efficient of variation $16.5 \%$ at the 99th percentile), in accordance with the manuacturer's instructions. All staff responsible for undertaking these analyses received bespoke training to run the i-Stat assays.

In addition, patients also underwent central laboratory cTn testing, which formed part of the reference standard for the diagnosis of AMI. In order to ensure that participants underwent adequate reference standard investigations for AMI, sites were asked to confirm that their local practice was consistent with current national and international guidance. Specifically, sites were required to confirm that patients would undergo the following cTn testing:

- If a contemporary (not high sensitivity) troponin assay was used: Laboratory-based troponin testing on arrival and either 6 hours after arrival or 10 to 12 hours after the onset of peak symptoms $[11,12]$ 
- If a high sensitivity troponin assay was used: Laboratory-based troponin testing on arrival and either 3 hours after arrival or 10 to 12 hours after the onset of peak symptoms [11,12] A high sensitivity troponin assay was defined as an assay that can detect troponin concentrations in at least $50 \%$ of apparently healthy individuals with a co-efficient of variation of $<10 \%$ at the 99 th percentile cut-off [13].

\section{Outcomes}

The primary outcome was the diagnosis of ACS. ACS was defined as either acute myocardial infarction (AMI), occurring during the initial hospital admission (prevalent $\mathrm{AMI}$ ), or incident major adverse cardiac events (MACE) occurring within 30 days. MACE included death (all cause), incident $\mathrm{AMI}$ and coronary revascularisation. All coronary revascularization procedures were considered to be relevant if they occurred within 30 days of the initial ED attendance. The diagnosis of AMI was allocated by two independent investigators, blinded to T-MACS and i-Stat cTnl concentrations. AMI was defined in accordance with the third universal definition of AMI [14] based on a rise and/or fall of cardiac troponin with at least one troponin concentration above the $99^{\text {th }}$ percentile of the assay, in conjunction with at least one of: symptoms of myocardial ischaemia, ECG changes or imaging evidence of new loss of viable myocardium. All relevant clinical notes and imaging reports were available for review by the adjudicators.

\section{Follow up}

Patients were followed up throughout their inpatient course and by telephone, email, letter or in person after 30 days. Data on length of stay; cardiac investigations and procedures; and details of any haemorrhagic complications were collected. If it was not possible to contact participants directly 
after repeated attempts, we obtained follow up information from patients' primary care practitioners where possible.

\section{Statistical analysis}

For the primary analyses, we evaluated the diagnostic accuracy of T-MACS used with point of care cTnl testing (i-Stat) at presentation and at 3 hours. For comparison, we also determined the diagnostic accuracy of the i-Stat cTnl assay when used alone (at presentation and after 3 hours) and in combination with ECG findings (as interpreted by the treating clinician), and we evaluated the diagnostic accuracy of T-MACS using the POC cTnl assay to the diagnostic accuracy when the central laboratory assay was used. For the latter evaluation, we used the cardiac troponin assay used in clinical practice. Four sites (Bolton, Harrogate, Northumbria and Basingstoke) were excluded from this analysis because the laboratory did not release cTn results at low concentrations, precluding calculation of T-MACS (which relies on the use of low cTn concentrations to identify 'very low risk' patients). Each of the remaining sites used the hs-cTnT assay (Roche Diagnostics Elecsys).

To evaluate diagnostic accuracy we calculated sensitivity, specificity, positive and negative predictive values (PPV and NPV), positive and negative likelihood ratios. We summarised the overall diagnostic accuracy of T-MACS and cTnl (i-Stat) by calculating the area under the receiver operating characteristic (ROC) curve. For these analyses, we excluded patients who did not have adequate reference standard investigations for $\mathrm{AMI}$, those who were lost to follow-up at 30 days and those who had missing data for T-MACS. Statistical analyses were completed in SPSS version 23.0 (SPSS Inc, Chicago, Illinois) and/or MedCalc version 13.1.2.0 (Mariakerke, Belgium).

To evaluate T-MACS, we applied the previously derived formula to estimate the probability of ACS, entering cTnl concentrations in $\mathrm{ng} / \mathrm{L}$ [7]. Consistent with our approach in the original model 
derivation, patients with cTnl concentrations below the limit of detection of the assay $(10 \mathrm{ng} / \mathrm{L})$ were considered to have concentrations of $9 \mathrm{ng} / \mathrm{L}$. For this evaluation, we used a minor modification to the original formula based on feedback from clinicians after implementation of the T-MACS algorithm. Clinicians had noted that patients with 'worsening (crescendo) angina' could be classified as 'low risk' (suitable for further evaluation in a low dependency inpatient environment) in the absence of other risk factors. However, they felt that such patients should be classified as 'moderate risk'. The coefficient for this variable was therefore manually re-calibrated to the minimum required to achieve this. Thus, the probability $(p)$ of ACS was calculated as follows:

$$
\mathrm{p}=1 /\left(1+\mathrm{e}^{-(-4.65+1.828 a+1.54 b+0.849 c+1.783 d+1.878 e+1.412 f+0.084 g)}\right)
$$

Where $a$ denotes acute ECG ischaemia; $b$ denotes a pattern of worsening (or crescendo) angina; $c$ is pain radiation to the right arm or right shoulder; $d$ is pain associated with vomiting; $e$ is visible diaphoresis in the ED; $f$ is hypotension (defined as systolic blood pressure $<100 \mathrm{mmHg}$ ); and $g$ is cTn concentration. For all variables except $g$, a value of ' 1 ' is entered if the feature is present and a value of ' 0 ' is entered if it is absent.

The T-MACS model classifies patients into four distinct risk groups based on their calculated risk probability according to the cut offs applied in the derivation of the original MACS rule. The four risk groups with associated suggestion for patient disposition include: (1) very low risk ( $p<0.02$; patients eligible for immediate discharge); (2) low risk $(0.02 \leq p<0.05$; suitable for serial cardiac troponin sampling in an ED observation ward or comparable alternative); (3) moderate risk ( $0.05 \leq p<0.95$; serial cardiac troponin sampling required in general ward such as an Acute Medical Ward); and (4) high risk ( $\mathrm{p} \geq 0.95$; ACS considered ruled in, best managed in a high dependency unit or specialist ward). 


\section{Sample size}

Assuming that the prevalence of the primary outcome was approximately $10 \%$, and that the algorithm would achieve $100 \%$ sensitivity, the lower bound of the $95 \% \mathrm{Cl}$ would be $>90 \%$ for sensitivity and $>99 \%$ for negative predictive value with a sample size of 605 participants. Accounting for potential loss to follow up and missing data (estimated to be approximately 5\%), we planned to include a total of approximately 650 participants. Recruitment was continued until we had verified data collection from all sites to ensure that this minimum sample size had been exceeded.

\section{Results}

We included a total of 716 patients at 8 centres, of which 105 (14.7\%) had ACS, including 89 (12.4\%) with prevalent AMI. During the recruitment period at participating centres, a total of 868 patients were recruited, although it was not possible to undertake the POC cTnl test for 126 patients because the analyser or cartridges were unavailable. This left 762 patients eligible for the analysis, of which 716 had full data and were included in the analysis of diagnostic accuracy (Figure 1). The baseline characteristics of participants are summarised in Table 1. A total of 634 patients underwent POC iStat testing at 3 hours, of which 97 (15.3\%) had ACS including 82 (12.9\%) with AMI. Based on a single i-Stat POC cTnl measurement at the time of arrival in the ED, the area under the reciever operating characteristic (ROC) curve (AUC) for T-MACS was 0.86 (95\% Cl $0.82-0.90)$. Accounting for the $3 \mathrm{~h}$ POC cTnl concentration increased the AUC to $0.92(95 \% \mathrm{Cl} 0.89-0.95)$.

The proportions of patients with ACS and AMI in each T-MACS risk group (based on i-Stat POC cTnI concentrations) are shown in Tables 2 and 3. Table 2 shows the proportions in each risk group based on a single POC cTnl test taken at the time of arrival in the ED. Table 3 shows the proportions based 
on two POC cTnl tests taken 3 hours apart. For the latter analysis, the maximum cTnl concentration detected was utilised.

\section{T-MACS as a 'rule out' test ('very low risk' vs all other risk groups)}

T-MACS could have been used to 'rule out' 306 (42.7\%) patients based on a single test at the time of arrival, or 196 (31.4\%) patients following a repeat cTnl test at 3h. Based on the initial POC cTnl concentration, there were six false negative results with T-MACS, including 4 patients with prevalent AMI and two patients who developed MACE within 30 days (both MACEs were percutaneous coronary intervention, $\mathrm{PCl})$.

Of those patients, only one remained 'false negative' once the second POC cTnl concentration measured at 3 hours had been taken into account. That patient did not have prevalent AMI (high sensitivity cTnT concentrations $4 \mathrm{ng} / \mathrm{L}$ and $5 \mathrm{ng} / \mathrm{L}$ respectively) but underwent invasive coronary angiography and $\mathrm{PCl}$ as an outpatient following discharge from hospital. The test characteristics of TMACS using POC cTnl on arrival and at 3 hours are shown in Table 4.

For comparison, if patients were 'ruled out' based on a single POC cTnl concentration $<10 \mathrm{ng} / \mathrm{L}$ on arrival and the absence of acute ECG ischaemia without accounting for T-MACS, a sensitivity of $87.4 \%$ (95\% $\mathrm{Cl} 79.4-93.1 \%)$ and NPV of $97.0 \%$ (95\% Cl 95.0 - 98.2\%) could have been achieved, and ACS would have been immediately 'ruled out' in $426(60.4 \%)$ patients. Similarly, accounting for the $3 \mathrm{~h}$ cTnl concentration with this strategy would have achieved a sensitivity of $93.7 \%(95 \% \mathrm{Cl} 86.8 \%$ 97.7\%) and an NPV of 98.3\% (95\% Cl 96.3-99.2\%), 'ruling out' 348 (55.8\%) patients. 


\section{T-MACS as a 'rule in' test ('high risk' vs all other risk groups)}

T-MACS could have 'ruled in' ACS in 49 (6.8\%) patients using the initial cTnl concentration with a PPV of $89.8 \%$ ( $95 \% \mathrm{Cl} 78.1-95.6 \%)$ and specificity $99.2 \%$ (95\% Cl $98.1-99.7 \%)$. In comparison, measuring POC cTnl concentration on arrival alone, with the $99^{\text {th }}$ percentile cut-off (80ng/L), could have 'ruled in' ACS for 42 (6.0\%) patients. This would have achieved a PPV of 90.5\% (95\% $77.6-$ $96.3 \%)$ with a specificity of $99.3 \%(95 \% \mathrm{Cl} 98.3-99.8 \%)$.

Also accounting for POC cTnl concentrations measured at 3 hours, T-MACS could have 'ruled in' ACS for $68(10.7 \%)$ patients. This achieved a PPV of $91.2 \%(95 \% \mathrm{Cl} 82.1-95.9 \%)$ and a specificity of $98.9 \%$ (95\% Cl 97.6 -99.6\%). Using POC cTnl concentrations alone with the $99^{\text {th }}$ percentile cut-off (80ng/L, considering the maximum concentration measured at presentation and 3 hours and without T-MACS) would have 'ruled in' ACS for a similar proportion of patients (10.4\%) with similar test characteristics (PPV 92.3\% and specificity 99.1\%).

\section{Diagnostic accuracy of T-MACS a central laboratory assay}

A total of 565 patients were included in this analysis, of which $65(11.5 \%)$ had AMI and $78(13.8 \%)$ had ACS. T-MACS identified 267 (47.2\%) patients as 'very low risk' with the central laboratory (hsCTnT) assay. Of those, two patients with AMI were wrongly identified as being 'very low risk' using the central laboratory assay and three had ACS. This gave a sensitivity of $98.9 \%$ ( $95 \% \mathrm{Cl} 96.8-99.8 \%)$ and an NPV of $98.6 \%(95 \% \mathrm{Cl} 95.6-99.6 \%)$ for ACS. For 'ruling in' ACS by identifying 30 (5.3\%) patients as 'high risk', T-MACS had a specificity of $99.4 \%$ (95\% Cl 98.2\% - 99.9\%) and PPV 90.0\% (95\% Cl 73.7-96.7\%). 


\section{Diagnostic accuracy of the POC cTn assay alone}

Without T-MACS, the POC cTn assay alone (tested at 0 \& $3 \mathrm{~h}$ using the $99^{\text {th }}$ percentile cut-off) had a sensitivity of $63.9 \%$ (95\% Cl 53.5 - 73.4\%), specificity $99.2 \%$ (95\% Cl 98.2 - 99.8\%), PPV 92.5\% (83.696.8\%) and NPV 94.8\% (93.3 - 95.9\%). If only patients with no ECG ischaemia were 'ruled out', test characteristics were as follows: sensitivity $73.2 \%$ (95\% Cl 63.2 - 81.7\%), specificity $93.1 \%$ (90.695.1\%), PPV 65.7\% (57.9 - 72.8\%) and NPV 95.1\% (93.3-96.4\%).

\section{Discussion}

In this work we have achieved two important goals with significant implications for practice. Our findings have identified wider clinical applications for (a) the POC cTnl i-Stat assay; and (b) the TMACS decision aid. By using the i-Stat cTnl assay alongside T-MACS, ACS could be 'ruled out' with serial sampling over 3 hours. Until now, guidelines have stated that the 3-hour rule out pathway should be reserved for use with high sensitivity cTn assays $[11,12,15]$. Our work suggests that the same can be achieved with a contemporary, POC assay, when used alongside the T-MACS decision aid.

In addition to 'ruling out' ACS, the algorithm could also enable the diagnosis to be 'ruled in' with over $90 \%$ positive predictive value, thus facilitating early access to specialist care for patients who will benefit the most. This compares very favourably to existing rapid 'rule in' algorithms. For example, using troponin criteria alone the PPV of a single test has been reported to be less than $90 \%$, even at very high cut-offs [16]. Even with serial sampling over 1 hour, the 1-hour rule-in and rule-out algorithm achieves a PPV of less than $80 \%$ [17]. These are not direct comparisons, and our work therefore does not suggest that the T-MACS is superior to these alternatives. However this other work does emphasise the value of achieving a PPV $>90 \%$, as reported here. 
Until now, the T-MACS decision aid had only been validated for use with high sensitivity cardiac troponin T (Roche) $[7,18]$ and contemporary (cardiac troponin I, Siemens cTnI-Ultra) [9] laboratorybased assays. Validation of the model with a POC cTnl assay enhances the possibilities for future clinical application. These possibilities include (a) expedited diagnostic evaluation in the ED, helping to reduce crowding; (b) enabling the use of biomarker testing in ambulatory care environments without a central laboratory on-site (for example, urgent care centres); and (c) diagnostic evaluation in the pre-hospital environment, including in the ambulance. The latter will require another prospective clinical study to establish the feasibility of using POC cTnl assays alongside the T-MACS decision aid in the pre-hospital environment. Because the algorithm is likely to be used sooner after symptom onset, it will also be important to verify its diagnostic accuracy in that environment. The Pre-hospital Evaluation of Sensitive Troponin (PRESTO) study, led by members of our group, will shortly address that objective.

Importantly, to obtain the benefits offered by POC testing, use of the accompanying T-MACS algorithm (which takes account of additional clinical information) is required to achieve sufficient diagnostic accuracy. Using POC cTnl concentrations alone, even with an unconventional 'rule out' cut-off at the extreme of the reportable range of the assay, could not 'rule out' ACS. These findings are entirely consistent with previous work which has shown that POC cTn assays used alone have suboptimal sensitivity $[19,20]$.

Our findings are also consistent with previous research evaluating other rapid rule out strategies using point of care biomarker assays in the Emergency Department. In the Randomised Assessment of Treatment using Panel Assay of Cardiac markers (RATPAC) study, who were randomised to receive point of care biomarker (cTn, myoglobin and creatine kinase MB [CK-MB] fraction) testing over 90 
minutes were more likely to be successfully discharged from the ED within 4 hours of arrival than patients who received central laboratory testing [21]. However, the strategy was not found to be cost-effective (possibly caused by over-triage relating to the use of non-specific biomarkers) [22] and the diagnostic accuracy of the point of care biomarkers (including cTn measured using the Siemens Stratus CS assay) was found to be inferior to central laboratory assays when used alone [23]. In Australasia, serial testing for cTn, myoglobin and CK-MB over 2 hours was found to rule out ACS with high sensitivity when used alongside the Thrombolysis in Myocardial Infarction (TIMI) risk score [24]. However, the strategy only identified $9.8 \%$ patients as eligible for early discharge, whereas using a central laboratory cTn assay maintained sensitivity while identifying $20 \%$ of patients as eligible for early discharge [3]. This current work adds to the literature by identifying that point of care cTn testing used alongside the T-MACS decision aid could identify over $30 \%$ patients as eligible for early discharge, while also 'ruling in' the diagnosis in other patients with high specificity.

\section{Limitations}

Although this is a multi-centre study at eight EDs and our total sample size of 716 patients exceeded the calculated requirement, our $95 \% \mathrm{Cls}$ were sufficiently wide to incorporate values that, if true, are unlikely to be clinically acceptable. Therefore, further prospective confirmation of our findings is desirable. We should also note that 126 patients did not undergo POC cTnl testing during the study period, due to a lack of available analysers or cTnl cartridges. A smaller number of patients had insufficient data recorded to calculate T-MACS or to verify the final diagnosis. It seems unlikely that this would substantially affect the results of our study as there is no suggestion that the missing data would introduce a systematic source of bias. Finally, we should acknowledge that while sites were encouraged to recruit a consecutive sample of patients, recruitment was ultimately dictated by researcher (predominantly research nurse) availability, meaning that this is ultimately a convenience sample. 
One key advantage of POC cTn assays is that the turnaround time is faster than central laboratory assays. In this work, it was not possible to quantify the time saving as our objective was to evaluate diagnostic accuracy. Thus, the POC tests were predominantly undertaken by research nurses who also had other non-clinical tasks to complete (such as seeking consent). Future work evaluating the implementation of POC cTn tests in practice should therefore seek to quantify the potential time saving when POC cTn assays are used.

The point of care troponin assays were also run by clinical research nurses and clinicians who had been received all appropriate study training and had been delegated responsibility to undertake the assays by the local Principal Investigator at each site. While this was required for governance reasons, and while the staff running the analyses have a similar background to all other clinical staff working in the ED, it will be important for future research to evaluate the assay when used as part of routine clinical practice.

\section{Conclusion}

The T-MACS decision aid could be used to 'rule in' and 'rule out' ACS with the POC cTnl i-Stat assay with serial samples drawn 3 hours apart. This would enable expedited diagnostic evaluation in EDs and may facilitate future use of both T-MACS and POC cTnl testing in ambulatory care and prehospital environments. 


\section{References}

1 Hospital Episode Statistics. Hospital admitted patient care activity, 2016-17: diagnosis.

2017.https://digital.nhs.uk/data-and-information/publications/statistical/hospital-admittedpatient-care-activity/2016-17 (accessed 19 Jun2018).

2 Body R, Carley S, McDowell G, Pemberton P, Burrows G, Cook G, et al. The Manchester Acute Coronary Syndromes (MACS) decision rule for suspected cardiac chest pain: derivation and external validation. Heart 2014;100:1462-8.

3 Than M, Cullen L, Aldous S, Parsonage WA, Reid CM, Greenslade J, et al. 2-Hour Accelerated Diagnostic Protocol to Assess Patients With Chest Pain Symptoms Using Contemporary Troponins as the Only Biomarker. Journal of the American College of Cardiology 2012;59:2091-8.

4 Pickering JW, Than MP, Cullen L, Aldous S, Ter Avest E, Body R, et al. Rapid Rule-out of Acute Myocardial Infarction With a Single High-Sensitivity Cardiac Troponin T Measurement Below the Limit of Detection: A Collaborative Meta-analysis. Ann Intern Med 2017;166:715-24.

5 Carlton E, Greenslade J, Cullen L, Body R, Than M, Pickering JW, et al. Evaluation of HighSensitivity Cardiac Troponin I Levels in Patients With Suspected Acute Coronary Syndrome. JAMA Cardiology 2016;1:405.

6 Van Den Berg P, Body R. The HEART score for early rule out of acute coronary syndromes in the emergency department: a systematic review and meta-analysis. Eur Heart J Acute Cardiovasc Care 2018;7:111-9.

7 Body R, Carlton E, Sperrin M, Lewis PS, Burrows G, Carley S, et al. Troponin-only Manchester Acute Coronary Syndromes (T-MACS) decision aid: single biomarker re-derivation and external validation in three cohorts. Emergency Medicine Journal 2017;34:349-56. 
8 Wu AHB, Christenson RH, Greene DN, Jaffe AS, Kavsak PA, Ordonez-Llanos J, et al. Clinical Laboratory Practice Recommendations for the Use of Cardiac Troponin in Acute Coronary Syndrome: Expert Opinion from the Academy of the American Association for Clinical Chemistry and the Task Force on Clinical Applications of Cardiac Bio-Markers of the International Federation of Clinical Chemistry and Laboratory Medicine. Clinical Chemistry 2018;64:645-55.

9 Van Den Berg P, Burrows G, Lewis P, Carley S, Body R. Validation of the (Troponin-only) Manchester Acute Coronary Syndromes decision aid with a contemporary cardiac troponin I assay. Am J Emerg Med 2018;36:602-7.

10 Amsterdam EA, Wenger NK, Brindis RG, Casey DE, Ganiats TG, Holmes DR, et al. 2014 AHA/ACC guideline for the management of patients with non-ST-elevation acute coronary syndromes: executive summary: a report of the American College of Cardiology/American Heart Association Task Force on Practice Guidelines. Circulation 2014;130:2354-94.

11 National Clinical Guideline Centre for Acute and Chronic Conditions. Chest pain of recent onset: NICE Clinical Guideline CG95. 2010.https://www.nice.org.uk/guidance/cg95 (accessed 20 Jun2018).

12 Roffi M, Patrono C, Collet J-P, Mueller C, Valgimigli M, Andreotti F, et al. 2015 ESC guidelines for the management of acute coronary syndromes in patients presenting without persistent STsegment elevation. Eur Heart J 2016;37(3):267-315.

13 Apple FS, Collinson PO, for the IFCC Task Force on Clinical Applications of Cardiac Biomarkers. Analytical Characteristics of High-Sensitivity Cardiac Troponin Assays. Clinical Chemistry 2011;58:54-61.

14 Thygesen K, Alpert JS, Jaffe AS, Simoons ML, Chaitman BR, White HD, et al. Third universal definition of myocardial infarction. Eur Heart J 2012;33:2551-67. 
15 NICE Diagnostics Guidance DG15. Myocardial infarction (acute): Early rule out using highsensitivity troponin tests (Elecsys Troponin T high-sensitive, ARCHITECT STAT High Sensitive Troponin-I and AccuTnl+3 assays). 2014.https://www.nice.org.uk/guidance/dg15 (accessed 13 Feb2015).

Mueller-Hennessen M, Mueller C, Giannitsis E, Biener M, Vafaie M, deFilippi CR, et al. Serial Sampling of High-Sensitivity Cardiac Troponin T May Not Be Required for Prediction of Acute Myocardial Infarction Diagnosis in Chest Pain Patients with Highly Abnormal Concentrations at Presentation. Clin Chem 2017;63:542-51.

17 Mueller C, Giannitsis E, Christ M, Ordóñez-Llanos J, deFilippi C, McCord J, et al. Multicenter Evaluation of a 0-Hour/1-Hour Algorithm in the Diagnosis of Myocardial Infarction With HighSensitivity Cardiac Troponin T. Annals of Emergency Medicine Published Online First: January 2016. doi:10.1016/j.annemergmed.2015.11.013

18 Greenslade JH, Nayer R, Parsonage W, Doig S, Young J, Pickering JW, et al. Validating the Manchester Acute Coronary Syndromes (MACS) and Troponin-only Manchester Acute Coronary Syndromes (T-MACS) rules for the prediction of acute myocardial infarction in patients presenting to the emergency department with chest pain. Emergency Medicine Journal 2017;34:517-23.

19 Palamalai V, Murakami MM, Apple FS. Diagnostic performance of four point of care cardiac troponin I assays to rule in and rule out acute myocardial infarction. Clinical Biochemistry 2013;46:1631-5.

20 Bruins Slot MHE, van der Heijden GJMG, Stelpstra SD, Hoes AW, Rutten FH. Point-of-care tests in suspected acute myocardial infarction: A systematic review. International Journal of Cardiology 2013;168:5355-62. 
21 Goodacre SW, Bradburn M, Cross E, Collinson P, Gray A, Hall AS, et al. The Randomised Assessment of Treatment using Panel Assay of Cardiac Markers (RATPAC) trial: a randomised controlled trial of point-of-care cardiac markers in the emergency department. Heart 2011;97:190-6.

22 Fitzgerald P, Goodacre SW, Cross E, Dixon S. Cost-effectiveness of point-of-care biomarker assessment for suspected myocardial infarction: the randomized assessment of treatment using panel assay of cardiac markers (RATPAC) trial. Acad Emerg Med 2011;18:488-95.

23 Collinson P, Gaze D, Goodacre S. Comparison of contemporary troponin assays with the novel biomarkers, heart fatty acid binding protein and copeptin, for the early confirmation or exclusion of myocardial infarction in patients presenting to the emergency department with chest pain. Heart 2014;100:140-5.

24 Than, M, Cullen, L, Reid, CM, Swee, HL, Aldous, S, Ardagh, MW, et al. A 2-h diagnostic protocol to assess patients with chest pain symptoms in the Asia-Pacific region (ASPECT): a prospective observational validation study. The Lancet 2011;377:1077-84. 


\section{Legends to figures}

Figure 1: Flow chart of study participants 


\section{Tables}

Table 1: Baseline characteristics of included patients

\begin{tabular}{|c|c|c|c|}
\hline & $\begin{array}{c}\text { Total } \\
(n=716)\end{array}$ & $\begin{array}{l}\text { ACS present } \\
\qquad(n=105)\end{array}$ & $\begin{array}{l}\text { ACS absent } \\
(n=611)\end{array}$ \\
\hline Age in years, mean (SD) & $57.6(15.6)$ & $66.1(14.6)$ & $56.2(15.3)$ \\
\hline Men (\%) & $445(62.2)$ & $78(74.3)$ & $367(60.1)$ \\
\hline Previous myocardial infarction (\%) & $169(23.6)$ & $35(33.3)$ & 134 (21.9) \\
\hline $\begin{array}{l}\text { Previous percutaneous coronary } \\
\text { intervention (\%) }\end{array}$ & $138(19.3)$ & $25(23.8)$ & $113(18.5)$ \\
\hline $\begin{array}{l}\text { Previous coronary artery bypass } \\
\text { graft }(\%)\end{array}$ & $51(7.1)$ & $12(11.4)$ & $39(6.4)$ \\
\hline Hypertension (\%) & $332(46.4)$ & $59(56.2)$ & $273(44.7)$ \\
\hline Hyperlipidaemia (\%) & $256(35.8)$ & $48(45.7)$ & $208(34.0)$ \\
\hline Type 1 diabetes mellitus (\%) & $14(2.0)$ & $4(3.8)$ & $10(1.6)$ \\
\hline Type 2 diabetes mellitus (\%) & $123(17.2)$ & $31(29.5)$ & $92(15.1)$ \\
\hline Current smoking (\%) & $139(19.4)$ & $28(26.7)$ & $111(18.2)$ \\
\hline $\begin{array}{l}\text { Time from symptom onset to } \\
\text { arrival in the ED: }\end{array}$ & & & \\
\hline $0-3 h$ & $354(49.4)$ & $54(51.4)$ & $300(49.1)$ \\
\hline $3-6 h$ & $153(21.4)$ & $25(23.8)$ & $128(20.9)$ \\
\hline $6-9 h$ & $88(12.3)$ & $14(13.3)$ & $74(12.1)$ \\
\hline$>9 h$ & $73(10.2)$ & $9(8.6)$ & $64(10.5)$ \\
\hline nents of the (T-)MACS rule & & & \\
\hline
\end{tabular}


Page $\mathbf{2 4}$ of $\mathbf{2 6}$

\begin{tabular}{|l|c|c|c|}
\hline Acute ECG ischaemia (\%) & $64(8.9)$ & $29(27.6)$ & $35(5.7)$ \\
\hline Worsening angina (\%) & $124(17.3)$ & $33(31.4)$ & $91(14.9)$ \\
\hline Pain associated with vomiting (\%) & $38(5.3)$ & $6(5.7)$ & $32(5.2)$ \\
\hline Sweating observed (\%) & $39(5.4)$ & $7(6.7)$ & $32(5.2)$ \\
\hline $\begin{array}{l}\text { Systolic blood pressure <100mmHg } \\
\text { (\%) }\end{array}$ & $23(3.2)$ & $3(2.9)$ & $20(3.3)$ \\
\hline Pain radiating to right arm or & $63(8.8)$ & $15(14.3)$ & $48(7.9)$ \\
shoulder (\%) & & & $173(28.3)$ \\
\hline POC cTnl (i-Stat) $\geq 10 \mathrm{ng} / \mathrm{L}$ (\%) & $263(36.7)$ & $90(85.7)$ & \\
\hline
\end{tabular}


Table 2: Proportion of patients with ACS and AMI in the four risk groups for the T-

MACS model (test on arrival only)

\begin{tabular}{|l|c|c|c|c|}
\hline & Very low risk & Low risk & Moderate risk & High risk \\
\hline Total number of patients & $306(42.7)$ & $134(18.7)$ & $227(31.7)$ & $49(6.8)$ \\
\hline Number (\%) with ACS & $6(2.0)$ & $16(11.9)$ & $39(17.2)$ & $44(89.8)$ \\
\hline Number (\%) with AMI & $4(1.3)$ & $16(12.3)$ & $26(11.6)$ & $43(87.8)$ \\
\hline
\end{tabular}

Table 3: Proportion of patients with ACS and AMI in the four risk groups for the T-

MACS model (test on arrival and at 3 hours)

\begin{tabular}{|l|c|c|c|c|}
\hline & Very low risk & Low risk & Moderate risk & High risk \\
\hline Total number of patients & $198(31.2)$ & $159(25.1)$ & $209(33.0)$ & $68(10.7)$ \\
\hline Number (\%) with ACS & $1(0.5)$ & $7(4.4)$ & $27(12.9)$ & $62(91.2)$ \\
\hline Number (\%) with AMI & $0(0.0)$ & $6(3.8)$ & $15(7.2)$ & $61(89.7)$ \\
\hline
\end{tabular}


Table 4: Diagnostic performance of the MACS and T-MACS models as 'rule out' strategies (i.e. 'very low risk' versus all other risk groups; 95\% confidence intervals in parentheses)

\begin{tabular}{|l|c|c|c|c|}
\hline \multirow{2}{*}{} & \multicolumn{2}{|c|}{ T-MACS, Oh only } & \multicolumn{2}{c|}{ T-MACS, Oh + 3h } \\
\cline { 2 - 5 } & For ACS & For AMI & For ACS & For AMI \\
\hline Sensitivity & 94.3 & 95.5 & 99.0 & 100.0 \\
& $(88.0-97.9)$ & $(88.9-98.8)$ & $(94.4-100.0)$ & $(95.6-100.0)$ \\
\hline Specificity & 49.1 & 48.2 & 36.7 & 35.9 \\
& $(45.1-53.1)$ & $(44.2-52.2)$ & $(32.6-40.9)$ & $(31.9-40.0)$ \\
\hline PPV & 24.2 & 20.7 & 22.0 & 18.8 \\
& $(22.5-25.9)$ & $(19.3-22.2)$ & $(20.9-23.2)$ & $(17.9-19.8)$ \\
\hline NPV & 98.0 & 98.7 & 99.5 & 100.0 \\
& $(95.8-99.1)$ & $(96.7-99.5)$ & $(96.5-99.9)$ & $($ NA) \\
\hline LR+ & 1.85 & 1.84 & 1.57 & $(1.47-1.66)$ \\
\hline LR- & $(1.69-2.03)$ & $(1.69-2.01)$ & $(1.46-1.68)$ & 0.00 \\
& 0.12 & 0.09 & 0.03 & $(0.00-0.20)$ \\
\hline
\end{tabular}

$\mathrm{PPV}=$ positive predictive value, $\mathrm{NPV}=$ negative predictive value, $\mathrm{LR}+=$ positive likelihood ratio, $\mathrm{LR}-=$ negative likelihood ration, $\mathrm{ACS}=$ acute coronary syndromes, $\mathrm{AMI}=$ acute myocardial infarction 